\title{
Optical diffraction tomography with fully and partially coherent illumination in high numerical aperture label-free microscopy
}

\author{
Juan M. Soto*, José A. Rodrigo and Tatiana Alieva ${ }^{1}$ \\ ${ }^{1}$ Universidad Complutense de Madrid, Facultad de Ciencias Físicas, Ciudad Universitaria s/n, Madrid 28040, Spain \\ *Corresponding author: juansoto@ucm.es \\ Compiled February 4, 2018
}

Quantitative label-free imaging is an important tool for the study of living micro organisms which during the last decade has attracted wide attention from the optical community. Optical diffraction tomography (ODT) is probably the most relevant technique for quantitative label-free 3D imaging applied in widefield microscopy in the visible range. The ODT is usually performed using spatially coherent light illumination and specially designed holographic microscopes. Nevertheless, the ODT is also compatible with partially coherent illumination and can be realized in conventional wide-field microscopes by applying refocusing techniques as it has been recently demonstrated. Here we compare these two ODT modalities underlining their pros and cons and discussing the optical setups for their implementation. In particular, we pay special attention to a system which is compatible with conventional wide-field microscope and can be used for both ODT modalities. It consists of two easily attachable modules: the first one for sample illumination engineering based on digital light processing technology and another one for focus scanning by using an electrically-driven tunable lens. This hardware allows for a programmable selection of the wavelength and the illumination design, as well as it provides fast data acquisition. Its performance is experimentally demonstrated in the case of ODT with partially coherent illumination providing specklefree 3D quantitative imaging.

(c) 2018 Optical Society of America

OCIS codes: (100.3010) Image reconstruction techniques; (030.0030) Coherence and statistical optics; (120.4630) Optical inspection; (170.6900) Three-dimensional microscopy; (110.4850) Optical transfer functions.

http://dx.doi.org/10.1364/ao.XX.XXXXXX

\section{INTRODUCTION}

The current trend of wide-field label-free microscopy of low absorption samples is not only obtaining high contrast images but also recovering from them quantitative information about the object structure (form, size or composition) directly related to its refractive index (RI) $n(\mathbf{r})$, where $\mathbf{r}=(x, y, z)^{t}$ is a $3 \mathrm{D}$ position vector. In general, thanks to the development of digital holography and computational optics, it has been possible to solve the inverse problem that aims at recovering information of the light scattered from a sequence of recorded intensity images. While this problem is relatively easy for thin two-dimensional (2D) objects it turns out to be much more complicated for thick objects (3D case), when tomographic methods need to be ap- plied. There are two approaches which are compatible with wide-field microscopy: computerized tomography (CT), also known as projection tomography, developed primary for X-ray optics, and the optical diffraction tomography (ODT) [1, 2]. Both of them were established in 50th-70th of the last century but their real applications in microscopy have started only after the development of proper optoelectronic devices, powerful computers and efficient signal processing algorithms. Their common idea is to illuminate the object from different sides. While, the difference between the CT [3-5] and the ODT [6-12] lies in the posterior processing of the measured intensity distributions of the light transmitted by the sample. The variation of the illumination angle in wide-field microscopy is usually achieved by scanning point by point the condenser aperture diaphragm 
$[3,4]$. Thus the sample is sequentially illuminated by spatially coherent plane waves propagated in different directions. The larger the condenser and objective numerical apertures (NAs), the broader the angular interval allowed for the object inspection. However, in wide-field microscopy not all the illumination directions are accessible and therefore it yields incomplete reconstruction of the 3D object spectrum (the well-known missing cone problem). In some cases this problem can be solved by rotating the object, by using a motorized rotation stage, see for example $[5,13,14]$. Alternatively, the artifacts arising from the missing cone problem can be mitigated by applying iterative post processing algorithms [15-17].

In the CT framework the eikonal approximation is assumed. In this case, from the phase of the optical field - recovered by interferometric, transport of intensity equation (TIE), iterative or any other method - the object thickness is obtained along each possible illumination direction. Later, the back filtered projection or other tomographic algorithms are applied to recover the distribution of the relative refractive index $\Delta n(\mathbf{r})=n(\mathbf{r})-n_{m}$ of the $3 \mathrm{D}$ object [4], where $n_{m}$ is the RI of the surrounding medium in which it is immersed. However, the eikonal approximation is only valid when the size $(l)$ of the inhomogeneities of the RI is significantly larger than the wavelength $\lambda$ and the width of the sample $L$ satisfies the following condition $\sqrt{\lambda L} \ll l$, that for visible wavelength range means approximately $l>5 \mu \mathrm{m}$ and $L \ll 50 \mu \mathrm{m}$. Therefore, it can not be used for analysis of object details of around $1 \mu \mathrm{m}$ size. In contrast, the ODT [6-12, 18, 19] takes into account the diffraction phenomena that in high NA microscopy allows for inspecting smaller objects than in the CT $[20,21]$. Instead of illuminating the object from different sides sequentially, it also can be done simultaneously using partially coherent illumination over the sample. In this case, an extended incoherent light source is located in the conjugated plane of the fully opened condenser diaphragm.

Here, we consider two different approaches for the realization of ODT using fully coherent illumination, hereinafter referred to as C-ODT, and partially coherent illumination (PC-ODT) and discuss their advantages and weaknesses. The paper is organized as follows. First we recall the fundamentals of the ODT and compare their modalities. Then we consider experimental setups suitable for C-ODT and PC-ODT implementation. Moreover, we propose an optical setup suitable for both ODT techniques. Next, since the vast majority of the literature is devoted to C-ODT, here an experimental demonstration of the PC-ODT performance is presented. The article ends with concluding remarks.

\section{ODT MODALITIES}

Let us consider the propagation of a monochromatic wave through a weakly absorption sample in wide-field transmission microscope. The ODT [1,2] was established based on small field perturbation method (e.g. see [22]) to resolve the scalar Helmholtz equation

$$
\left(\nabla^{2}+k^{2}(\mathbf{r})\right) u(\mathbf{r})=0,
$$

for the propagation of the coherent optical wave in an inhomogeneous medium characterized by its RI, $n(\mathbf{r})$. Here, $u(\mathbf{r})$ is a complex field amplitude and $k(\mathbf{r})=k_{0} n(\mathbf{r})=2 \pi n(\mathbf{r}) / \lambda_{0}$ is a wavenumber with $\lambda_{0}$ being the light wavelength in vacuum. By introducing the RI of the surrounding medium $n_{m}$, the previous equation can be rewritten as

$$
\left(\nabla^{2}+k_{m}^{2}\right) u(\mathbf{r})=-V(\mathbf{r}) u(\mathbf{r})
$$

where $V(\mathbf{r})=k_{0}^{2}\left(n^{2}(\mathbf{r})-n_{m}^{2}\right)$ is the so called scattering potential and $k_{m}=k_{0} n_{m}$. From the knowledge of the solution $u_{0}(\mathbf{r})$ for the homogeneous Helmhotz equation $(V(\mathbf{r})=0)$ and Green's function $G(\mathbf{r})=-\exp \left(\mathrm{i} k_{m}|\mathbf{r}|\right) /(4 \pi|\mathbf{r}|)$, which satisfies the Helmhotz equation for a point-like source $\delta\left(\mathbf{r}-\mathbf{r}^{\prime}\right)$, the solution of the Eq. (2) can be written in the integral form as

$$
u(\mathbf{r})=u_{0}(\mathbf{r})+\int G\left(\mathbf{r}-\mathbf{r}^{\prime}\right) V\left(\mathbf{r}^{\prime}\right) u\left(\mathbf{r}^{\prime}\right) \mathrm{d} \mathbf{r}^{\prime} .
$$

Writing the complex field amplitude as a linear superposition $u(\mathbf{r})=u_{0}(\mathbf{r})+u_{s}(\mathbf{r})$, where $u_{0}(\mathbf{r})$ and $u_{s}(\mathbf{r})$ respectively describe the incident and scattered light, and using the weakscattering condition $\left(\left|u_{s}\right| \ll\left|u_{0}\right|\right)$, the integral term in Eq. (3) is transformed into a convolution operation:

$$
u_{s}(\mathbf{r}) \stackrel{\text { Born }}{=} \int G\left(\mathbf{r}-\mathbf{r}^{\prime}\right) V\left(\mathbf{r}^{\prime}\right) u_{0}\left(\mathbf{r}^{\prime}\right) \mathrm{d} \mathbf{r}^{\prime}=\left[V(\mathbf{r}) u_{0}(\mathbf{r})\right] * G(\mathbf{r}) .
$$

Then, the complex field amplitude of the optical field passing through the sample is given by

$$
u(\mathbf{r}) \stackrel{\text { Born }}{=} u_{0}(\mathbf{r})+\left[V(\mathbf{r}) u_{0}(\mathbf{r})\right] * G(\mathbf{r}) .
$$

Note that this first-order Born approximation only takes into account the undiffracted light and its interference with oncescattered light, thus excluding multiple scattering. Following this approximation the term $\left|u_{\mathcal{S}}(\mathbf{r})\right|^{2}$ in the intensity distribution $I(\mathbf{r})=|u(\mathbf{r})|^{2}$ is ignored:

$$
I(\mathbf{r})=|u(\mathbf{r})|^{2} \stackrel{\text { Born }}{=}\left|u_{0}(\mathbf{r})\right|^{2}+2 \operatorname{Re}\left\{u_{0}^{*}(\mathbf{r}) u_{s}(\mathbf{r})\right\} .
$$

Instead of Born approximation, Rytov approximation is often applied in ODT, e.g. see [15, 20, 23]. It assumes slow phase changing that implies a low refractive index gradient of the inhomogeneities and therefore is more suitable for the description of light interaction with relatively large and smooth objects. However, since here we consider the implementation of the ODT with high-numerical aperture optics enabling high resolution imaging of small object details, the Born approximation is a proper choice.

The realization of the C-ODT consists of the sequential illumination of the sample by using spatially-coherent plane waves, propagating in different directions, and the subsequent recovery of the complex field amplitude of the transmitted field for each illumination direction. Alternatively, object rotation can be used $[5,13,14]$, however, it is a challenging task for small living organisms. Conversely, the scanning illumination beam is easily achieved by the variation of the position of a laser spot onto the back focal plane of the condenser lens (e.g., see $[8,15])$. The larger the NA of the condenser lens, the wider is the angular interval available for object inspection.

Under plane-wave illumination $u_{0}(\mathbf{r} \mid \mathbf{s})=a(\mid \mathbf{s}) \exp \left(\mathrm{i} k_{m} \mathbf{s r}\right)$, where $\mathbf{s}=\left(s_{x}, s_{y}, s_{z}\right)=\left(s_{x}, s_{y}, \sqrt{1-s_{x}^{2}-s_{y}^{2}}\right)^{t}$ is the direction of the wave propagation and $a(\mid \mathbf{s})$ stands for its amplitude, the complex field amplitude passed through the sample is given by

$$
u(\mathbf{r} \mid \mathbf{s})=a(\mid \mathbf{s}) \exp \left(\mathrm{i} k_{m} \mathbf{s r}\right)+a(\mid \mathbf{s})\left[V(\mathbf{r}) \exp \left(\mathrm{i} k_{m} \mathbf{s r}\right)\right] * G(\mathbf{r})
$$

Note that here and further the symbol $\mid \mathbf{s}$ indicates the parameter associated with the considered illumination direction. The objective lens collects the transmitted waves thus yielding the convolution with its point spread function $h_{o}(\mathbf{r})$ as it follows: 


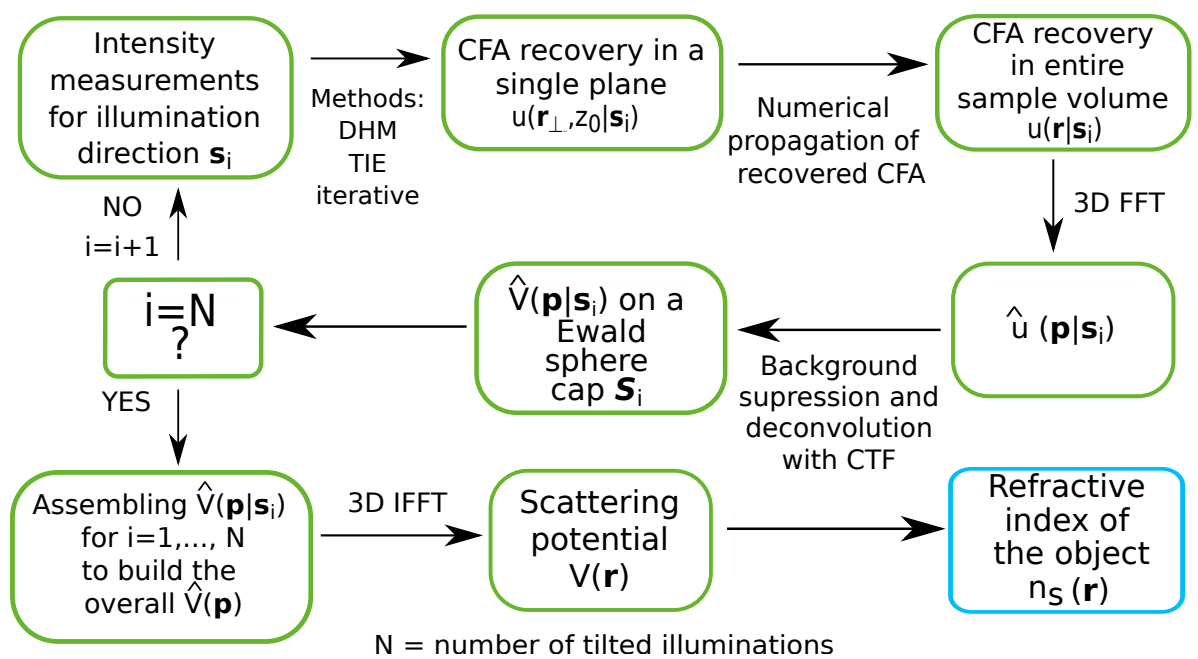

Fig. 1. Flowchart of the information processing approach used in C-ODT for reconstructing the 3D RI. From an intensity measurement for a certain illumination direction $\left(\mathbf{s}_{i}\right)$, the complex field amplitude (CFA) of the light transmitted by the microscope is retrieved at a single plane $z_{0}$. The type of intensity measurement (hologram(s), refocused images) depends on the method used for phase retrieval (DH, TIE, iterative algorithms). The CFA in the entire sample volume is obtained by numerical propagation of the retrieved CFA in $z_{0}$. Next, the 3D FT of the CFA is computed. Then, after a background suppression and a deconvolution procedure with the coherent transfer function $(\mathrm{CTF})$, the scattering potential into the corresponding cap of the Ewald sphere is recovered. This procedure is repeated by changing $\mathbf{s}_{i}$ for a predetermined number of illuminations $(\mathrm{N})$. Later, all the pieces of the scattering potential obtained separately are assembled together in the spectral domain. Finally, by using the 3D inverse FT (IFFT) operation, the scattering potential in the spatial domain and the RI are recovered.

$$
\begin{aligned}
u(\mathbf{r} \mid \mathbf{s}) & =a(\mid \mathbf{s})\left\{\exp \left(\mathrm{i} k_{m} \mathbf{s r}\right) * h_{o}(\mathbf{r})\right. \\
& \left.+\left[V(\mathbf{r}) \exp \left(\mathrm{i} k_{m} \mathbf{s r}\right)\right] * G(\mathbf{r}) * h_{o}(\mathbf{r})\right\}
\end{aligned}
$$

Calculating the 3D FT, $\widehat{u}(\mathbf{p} \mid \mathbf{s})=\int u(\mathbf{r} \mid \mathbf{s}) \exp \left(-\mathrm{i} 2 \pi \mathbf{r} \mathbf{p}^{t}\right) \mathrm{d} \mathbf{r}$, the Eq. (8) can be rewritten as:

$\widehat{u}(\mathbf{p} \mid \mathbf{s})=a(\mid \mathbf{s}) H_{o}(\mathbf{p}) \delta\left(\mathbf{p}-\kappa_{m} \mathbf{s}\right)+a(\mid \mathbf{s}) H_{o}(\mathbf{p}) \widehat{G}(\mathbf{p}) \hat{V}\left(\mathbf{p}-\kappa_{m} \mathbf{s}\right)$,

where $\mathbf{p}=\left(p_{x}, p_{y}, p_{z}\right)^{t}$ is a 3D spatial frequency vector, $2 \pi \kappa_{m}=$ $k_{m}$ and $H_{o}(\mathbf{p})$ is the transfer function of the objective lens. Indeed, by applying a digital Notch filtering for background (unscattered light) suppression, the spectrum of the scattering potential is derived:

$$
\hat{V}(\mathbf{p} \mid \mathbf{s})=\frac{\widehat{u}\left(\mathbf{p}+\kappa_{m} \mathbf{s} \mid \mathbf{s}\right)-a(\mid \mathbf{s}) H_{o}\left(\mathbf{p}+\kappa_{m} \mathbf{s}\right) \delta(\mathbf{p})}{a(\mid \mathbf{s}) H_{o}\left(\mathbf{p}+\kappa_{m} \mathbf{s}\right) \widehat{G}\left(\mathbf{p}+\kappa_{m} \mathbf{s}\right)} .
$$

Note that in the case of a microscope objective lens with a circular aperture its transfer function can be approximated by [24],

$$
H_{o}(\mathbf{p})=\operatorname{circ}\left(\frac{\lambda \sqrt{p_{x}^{2}+p_{y}^{2}}}{\mathrm{NA}_{\mathrm{o}}}\right) \operatorname{step}\left(\frac{p_{z}}{\kappa_{m}}\right),
$$

where $\mathrm{NA}_{\mathrm{O}}$ accounts for the numerical aperture of the objective lens. Alternatively, the coherent transfer function (CTF) $H_{o}(\mathbf{p}) \widehat{G}(\mathbf{p})$ can be experimentally measured $[7,8]$.

Since we consider an elastic processes $\left|\kappa_{m} \mathbf{s}\right|=\left|\mathbf{q}_{d}\right|=$ $\left|\mathbf{p}+\kappa_{m} \mathbf{s}\right|$, where $\mathbf{q}_{d}$ is the wave vector of the scattered light, then the accessible object frequencies lie into a cap of the Ewald sphere of radius $\kappa_{m}$ with its center at $\kappa_{m} \mathbf{s}$. Note that the angle subtended by this cap is defined by the NA of the objective lens. Then, after the deconvolution procedure given by Eq. (10) only a part of the scattering potential spectrum can be recovered. The variation of the incident wave directions yields a displacement of the center of the cap and therefore it permits enlarging the area of the spectral domain to be recovered. The proper assembling of the collected spectral components $\hat{V}(\mathbf{p} \mid \mathbf{s})$, recovered for different $\mathbf{s}$, in $\hat{V}(\mathbf{p})$ makes possible the reconstruction of the scattering potential via its $3 \mathrm{D}$ inverse FT. However, the limited angular coverage of the condenser lens aperture (usually is not extended more than $60^{\circ}$ with respect to the optical axis) yields the aforementioned missing cone problem responsible of the incomplete recovery of $\hat{V}(\mathbf{p})$. Consequently, several artifacts such as axial elongation are present in the reconstructed 3D RI distribution, which can be mitigated by using post-processing algorithms [15-17].

Note that the scattering potential is, in general, a complex valued function

$$
\begin{aligned}
& V(\mathbf{r})=P(\mathbf{r})+\mathrm{i} A(\mathbf{r}), \\
& P(\mathbf{r})=k_{0}^{2}\left(n_{\mathrm{re}}^{2}-n_{\mathrm{im}}^{2}-n_{m}^{2}\right), \\
& A(\mathbf{r})=2 k_{0}^{2} n_{\mathrm{re}} n_{\mathrm{im},}
\end{aligned}
$$

whose real and imaginary parts, $P(\mathbf{r})$ and $A(\mathbf{r})$ respectively, are related to the real $\left(n_{\mathrm{re}}\right)$ and imaginary $\left(n_{\mathrm{im}}\right)$ parts of the RI which can be easily found from $V(\mathbf{r})$. However, in ODT microscopy weak absorbing samples (negligible $n_{\text {im }}$ ) are usually considered, therefore it is $n_{\text {re }}$ which reveals the object structure.

As we have mentioned the C-ODT requires the knowledge of the complex-field amplitude of the object transmitted field. A scheme for 3D RI reconstruction for the case of C-ODT is displayed in Fig. 1. It starts with the acquisition of one or several intensity distributions by using a digital camera for a certain plane-wave illumination (collimated beam) for $\mathbf{s}_{i}$. When using a method based on digital holography (DH), the mentioned intensity distributions correspond to holograms from which 
(a) $\mathrm{N}=1$
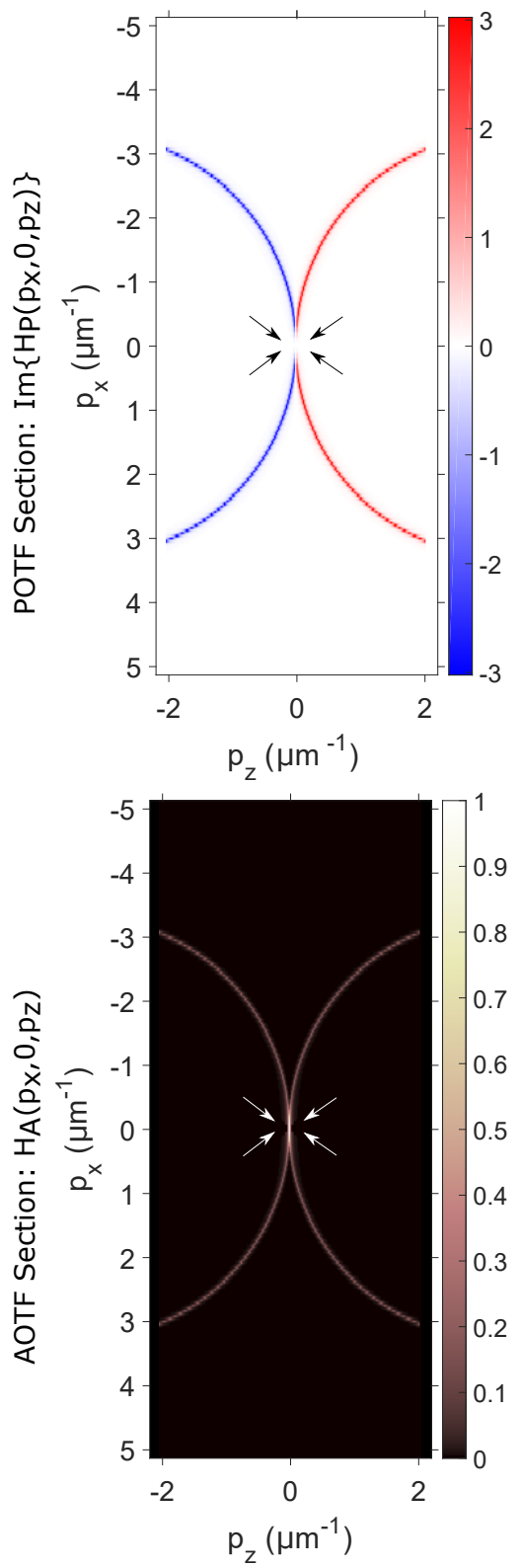

(b) $\mathrm{N}=10$
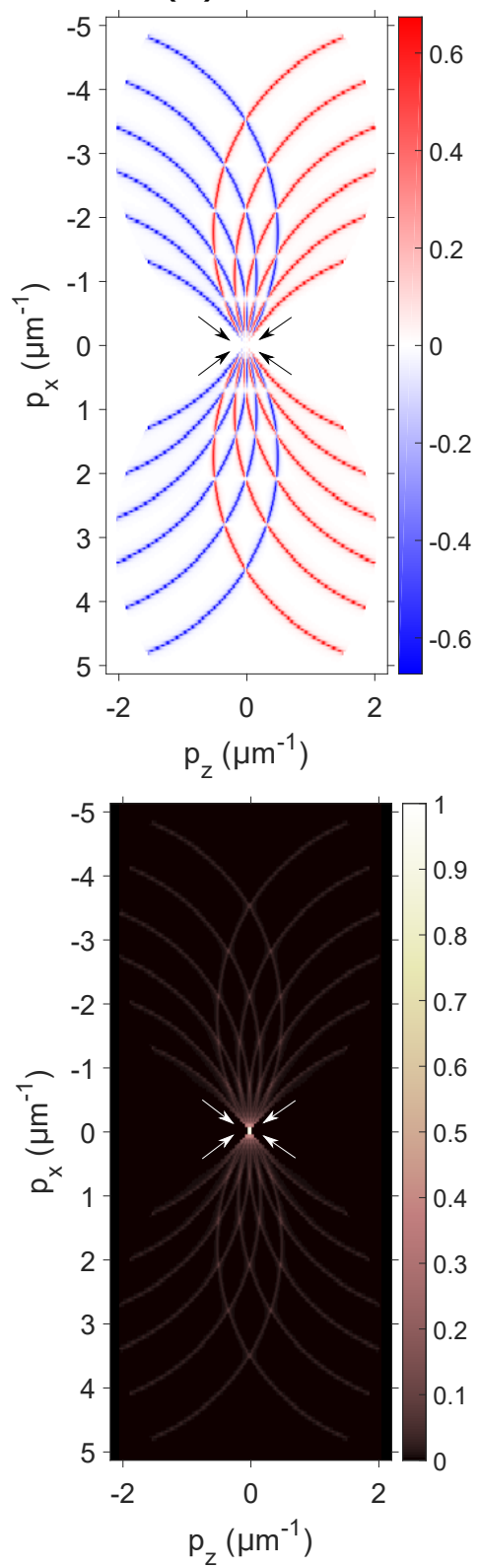

(c) $\mathrm{N} \rightarrow \infty$
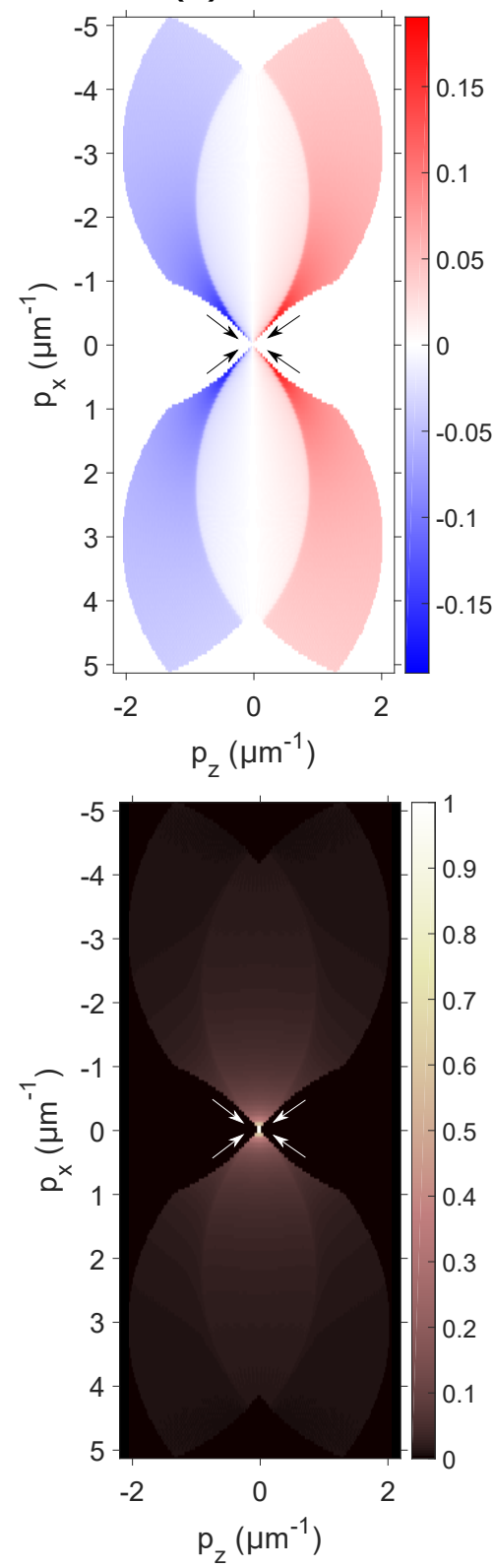

Fig. 2. Comparison between different $2 \mathrm{D} X Z$ sections of the $3 \mathrm{D}$ nonparaxial OTF. The first row summarizes different phase OTFs (POTFs), $H_{P}(\mathbf{p})$, whereas the second row shows the corresponding sections of the absorption transfer function $(\mathrm{AOTF}), H_{A}(\mathbf{p})$. OTFs from (a-c) columns have been calculated with a different number of illumination plane waves (N), ranging from an almost coherent situation (a) to a coherent regime close to incoherent illumination (c). All the OTFs have been represented by taking $\lambda_{0}=$ $450 \mathrm{~nm}, \mathrm{NA}_{\mathrm{c}}=0.95$ and $\mathrm{NA}_{\mathrm{o}}=1.4$. The black and white arrows indicate the missing-cone region in the frequency domain.

the required complex-field amplitudes can be retrieved. For instance, in the case of off-line holographic setups one hologram per tilted illumination is sufficient to retrieve the corresponding complex-field amplitude. On the other hand, in the case of on-line holography it is necessary to measure at least four holograms (by using phase-shifting setup) to retrieve each complexfield amplitude. Alternatively, TIE or iterative methods can be used instead of $\mathrm{DH}$, in which case such intensity distributions correspond to $4-10$ optically refocused images. The numerical refocusing of the retrieved complex-field amplitude allows for the recovery of a optical field in the entire sample volume. Then the 3D Fourier Transform (FT) of such a complex-field amplitude stack is calculated. The background suppression is applied at this step (or, alternatively, previously in the $\mathbf{r}$ domain). Note that the deconvolution described by Eq. (10) only provides the accessible content of the scattering potential spectrum $\hat{V}\left(\mathbf{p} \mid \mathbf{s}_{i}\right)$ lying on the captured cap of the Ewald sphere defined by $\mathbf{s}_{i}$ and $\mathrm{NA}_{\mathrm{o}}$. Afterwards, the described process is repeated for each illumination direction until reaching a predetermined number $(N)$. Ideally, the whole condenser aperture diaphragm of the optical system must be scanned point by point providing all the possible illumination directions. Nevertheless, in order to make more practical such a time-multiplexing approach, some researches have proposed a scanning reduced to spiral trajecto- 
ries [15] achieving a good trade-off between accuracy and speed of the RI reconstruction. Finally, all the computed pieces of the 3D scattering potential spectrum, $\hat{V}\left(\mathbf{p} \mid \mathbf{s}_{i}\right)$ (with $i=1, \ldots, N$ ) that have been obtained separately, are assembled together to build the overall 3D scattering potential spectra $\hat{V}(\mathbf{p})$. From the latter one, and after performing an inverse 3D FT (IFFT), one gets the scattering potential in the spatial domain, $V(\mathbf{r})$, from which the 3D RI image reconstruction is straightforward following the expression Eq. (12).

Alternatively, instead of the aforementioned deconvolution approach (see, Eq. (10)), the so-called filtered backpropagation algorithm (do not be confused with filtered backprojection algorithm used in CT [25]) can be used for the recovery of $V(\mathbf{r})$ in C-ODT. However, this is more appropriate for the case of object rotation [26, 27], while in the scheme considered here both the object and the detector plane are fixed and the 3D information is obtained by using the illumination rotation. Moreover, the deconvolution method considered here allows easily taking into account the microscope transfer function. Both of these C-ODT approaches can be applied for Born and Rytov approximation since the Rytov complex phase $\phi(\mathbf{r})=u_{s}(\mathbf{r}) / u_{0}(\mathbf{r})$ is directly related to the scattered complex-field amplitude for Born approximation, $u_{s}(\mathbf{r})$.

In the case of partially coherent illumination it is also possible retrieving the scattering potential and reconstructing the 3D RI. The theoretical fundamentals of PC-ODT technique have been established thirty years ago by N. Streibl [28] but its experimental realization has been started rather recently [23, 29, 30]. Instead of using a coherent laser beam, in PC-ODT the spatially incoherent quasi-monochromatic light available in conventional microscopes (either LED or filtered halogen lamp) is projected onto the back aperture of the condenser lens thus providing the object illumination from all the angles allowed in the C-ODT but simultaneously. Let us assume that the incoherent intensity distribution over the condenser aperture diaphragm is $a^{2}(\mid \mathbf{s})$. Then the measured intensity distribution is a sum of all the intensity contributions obtained by the coherent plane wave illumination at different angles. The intensity associated with the plane wave $u_{0}(\mathbf{r} \mid \mathbf{s})=a(\mid \mathbf{s}) \exp \left(\mathrm{i} k_{m} \mathbf{s r}\right)$ reaching the object from the direction $\mathbf{s}$ can be easily found from the previous considerations (see Eqs. (6)-(8)):

$$
\begin{gathered}
I(\mathbf{r} \mid \mathbf{s})=a^{2}(\mid \mathbf{s})\left[\left|\exp \left(\mathrm{i} k_{m} \mathbf{s r}\right) * h_{o}(\mathbf{r})\right|^{2}\right. \\
+\left[\exp \left(\mathrm{i} k_{m} \mathbf{s r}\right) * h_{o}(\mathbf{r})\right]^{*}\left[V(\mathbf{r}) \exp \left(\mathrm{i} k_{m} \mathbf{s r}\right)\right] * G(\mathbf{r}) * h_{o}(\mathbf{r}) \\
\left.+\left[\exp \left(\mathrm{i} k_{m} \mathbf{s r}\right) * h_{o}(\mathbf{r})\right]\left\{\left[V(\mathbf{r}) \exp \left(\mathrm{i} k_{m} \mathbf{s r}\right)\right] * G(\mathbf{r}) * h_{o}(\mathbf{r})\right\}^{*}\right],
\end{gathered}
$$

where the term $I_{0}(\mathbf{r} \mid \mathbf{s})=a^{2}(\mid \mathbf{s})\left|\exp \left(\mathrm{i} k_{m} \mathbf{s r}\right) * h_{o}(\mathbf{r})\right|^{2}=$ $a^{2}(\mid \mathbf{s})\left|H_{o}\left(\kappa_{m} \mathbf{s}\right)\right|^{2}$ denotes the DC term representing the background intensity. By expanding the scattering potential into its real $P(\mathbf{r})$ and imaginary $A(\mathbf{r})$ parts according with Eq. (12), the last two terms of the normalized intensity $J(\mathbf{r} \mid \mathbf{s})=$ $\left[I(\mathbf{r} \mid \mathbf{s})-I_{0}(\mathbf{r} \mid \mathbf{s})\right] / a^{2}(\mid \mathbf{s})$ can be rewritten as

$$
\begin{aligned}
& J(\mathbf{r} \mid \mathbf{s})=\left[(P(\mathbf{r})+\mathrm{i} A(\mathbf{r})) \exp \left(\mathrm{i} k_{m} \mathbf{s r}\right)\right] * G(\mathbf{r}) * h_{o}(\mathbf{r}) \\
& \quad \times\left[\exp \left(-\mathrm{i} k_{m} \mathbf{s r}\right) * h_{o}^{*}(\mathbf{r})\right]+\left[\exp \left(\mathrm{i} k_{m} \mathbf{s r}\right) * h_{o}(\mathbf{r})\right] \\
& \quad \times\left[(P(\mathbf{r})-\mathrm{i} A(\mathbf{r})) \exp \left(-\mathrm{i} k_{m} \mathbf{s r}\right)\right] * G^{*}(\mathbf{r}) * h_{o}^{*}(\mathbf{r}) .
\end{aligned}
$$

Afterwards, by taking the 3D FT on both sides of Eq. (14) one obtains:

$$
\begin{gathered}
\hat{J}(\mathbf{p} \mid \mathbf{s})=\hat{P}(\mathbf{p})\left[H_{o}^{*}\left(\kappa_{m} \mathbf{s}\right) \widehat{G}\left(\mathbf{p}+\kappa_{m} \mathbf{s}\right) H_{o}\left(\mathbf{p}+\kappa_{m} \mathbf{s}\right)\right. \\
\left.+H_{o}\left(\kappa_{m} \mathbf{s}\right) \widehat{G}^{*}\left(-\mathbf{p}+\kappa_{m} \mathbf{s}\right) H_{o}^{*}\left(-\mathbf{p}+\kappa_{m} \mathbf{s}\right)\right] \\
+\mathrm{i} \hat{A}(\mathbf{p})\left[H_{o}^{*}\left(\kappa_{m} \mathbf{s}\right) \widehat{G}\left(\mathbf{p}+\kappa_{m} \mathbf{s}\right) H_{o}\left(\mathbf{p}+\kappa_{m} \mathbf{s}\right)\right. \\
\left.-H_{o}\left(\kappa_{m} \mathbf{s}\right) \widehat{G}^{*}\left(-\mathbf{p}+\kappa_{m} \mathbf{s}\right) H_{o}^{*}\left(-\mathbf{p}+\kappa_{m} \mathbf{s}\right)\right] .
\end{gathered}
$$

Finally, after an integration over all possible illumination directions limited by the condenser aperture diaphragm $S(\mathbf{s})=\operatorname{circ}\left(\sqrt{s_{x}^{2}+s_{y}^{2}} / \mathrm{NA}_{\mathrm{c}}\right) \operatorname{step}\left(s_{z}\right)$, thus considering that plane waves coming from all possible directions are mutually incoherent, the 3D FT of the stack of intensity distributions at the sample region is given by

$$
\begin{aligned}
\widehat{I}(\mathbf{p}) & =\int_{S} \widehat{I}(\mathbf{p} \mid \mathbf{s}) \mathrm{d} \mathbf{s} \\
& =B \delta(\mathbf{p})+\hat{A}(\mathbf{p}) H_{A}(\mathbf{p})+\hat{P}(\mathbf{p}) H_{P}(\mathbf{p}) .
\end{aligned}
$$

Here, the term $B=\int_{S} I_{0}(\mathbf{r} \mid \mathbf{s})$ ds is the overall background intensity, $H_{A}(\mathbf{p})$ and $H_{P}(\mathbf{p})$ are the so-called absorption (AOTF) and phase (POTF) transfer functions [23, 28, 29] defined by

$$
\begin{array}{r}
H_{A}(\mathbf{p})=\mathrm{i} \int_{S} a^{2}(\mid \mathbf{s})\left[H_{o}^{*}\left(\kappa_{m} \mathbf{s}\right) \widehat{G}\left(\mathbf{p}+\kappa_{m} \mathbf{s}\right) H_{o}\left(\mathbf{p}+\kappa_{m} \mathbf{s}\right)\right. \\
\left.-H_{O}\left(\kappa_{m} \mathbf{s}\right) \widehat{G}^{*}\left(-\mathbf{p}+\kappa_{m} \mathbf{s}\right) H_{o}^{*}\left(-\mathbf{p}+\kappa_{m} \mathbf{s}\right)\right] \mathrm{d} \mathbf{s}, \\
H_{P}(\mathbf{p})=\int_{S} a^{2}(\mid \mathbf{s})\left[H_{o}^{*}\left(\kappa_{m} \mathbf{s}\right) \widehat{G}\left(\mathbf{p}+\kappa_{m} \mathbf{s}\right) H_{o}\left(\mathbf{p}+\kappa_{m} \mathbf{s}\right)\right. \\
\left.\quad+H_{o}\left(\kappa_{m} \mathbf{s}\right) \widehat{G}^{*}\left(-\mathbf{p}+\kappa_{m} \mathbf{s}\right) H_{o}^{*}\left(-\mathbf{p}+\kappa_{m} \mathbf{s}\right)\right] \mathrm{d} \mathbf{s} .
\end{array}
$$

It is easy to see that $H_{A}^{*}(\mathbf{p})=H_{A}(\mathbf{p})$ while the POTF is a Hermitian function $H_{P}^{*}(-\mathbf{p})=H_{P}(\mathbf{p})$. Consequently, the AOTF is a real even function whereas the imaginary part of the POTF is odd. In the case of uniform illumination, projected over the entire back aperture of the condenser lens, the term $a^{2}(\mid \mathbf{s})=a^{2}$ is independent on $\mathbf{s}$ and therefore the integrals in Eqs. (17) can be analytically calculated $[24,28]$. The contribution of illuminating plane waves in the total POTF and AOTF is illustrated in the first and the second row of Fig. 2, respectively. Specifically, Fig. 2 (a-c) correspond to the $\left(p_{x}, 0, p_{z}\right)$ sections of the OTFs for the following cases: in Fig. 2 (a) a plane wave provides coherent illumination (only the central point of the condenser aperture diaphragm has been illuminated, thus $\mathbf{s}=(0,0,1))$, while in Fig. 2 (b) and 2 (c) intermediate (with 10 different illumination directions) and almost incoherent (the whole condenser aperture diaphragm is filled with light) illumination have been considered, respectively. As it is observed in Fig. 2 the larger the number of illumination plane waves, the more spectrum region is covered.

While the scattering potential of phase-only (non-absorbing) objects $(A(\mathbf{r})=0)$ can be easily recovered from the intensity stack spectrum, the direct application of this method becomes complicated if the absorption of the object has to be taken into account. To separately reconstruct the real and the imaginary part of the $V(\mathbf{r})$, an additional axial intensity stack for a different type of illumination and therefore with different microscope transfer functions $H_{A}(\mathbf{p})$ and $H_{P}(\mathbf{p})$ has to be recorded. This 


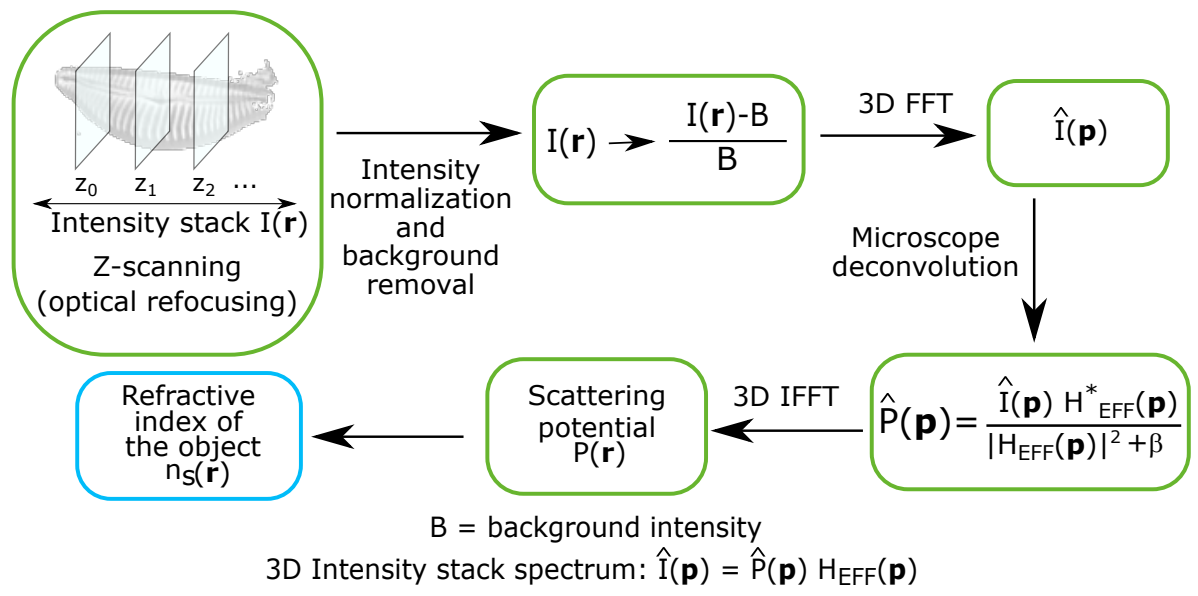

Fig. 3. Flowchart of the information processing approach used in PC-ODT for reconstruction of the object 3D RI. A 3D intensity stack $I(\mathbf{r})$ of refocused intensity images (in bright-field modality) is measured by axial scanning of the sample. Then, a intensity normalization is performed to impose energy conservation, followed by a background removal in $I(\mathbf{r})$. Next, a 3D FT of $I(\mathbf{r})$ is calculated. Later, $\hat{I}(\mathbf{p})$ is deconvolved by using the microscope effective transfer function in order to obtain the scattering potential in the spectral domain, $\hat{P}(\mathbf{p})$. Finally, the $3 \mathrm{D}$ inverse FT is applied to obtain the scattering potential in the spatial domain from which the RI of the sample is obtained.

can be achieved, for example, by variation of the illumination distribution $a^{2}(\mid \mathbf{s})$ over the condenser aperture diaphragm [28]. A similar approach has been studied in the context of differential contrast imaging modality that in principle allows for recovering of both the real and imaginary parts of the RI [29]. However, it increases the amount of information that needs to be acquired and processed, thus requiring much longer time for the 3D RI reconstruction. Another possibility is considering that the real and imaginary parts of the scattering potential fulfill the condition $A(\mathbf{r})=\varepsilon P(\mathbf{r})$, where $\varepsilon$ takes a small positive value (in the range $0-0.1$ for weak absorption objects, see [30]). This approach is equivalent to the introduction of an effective transfer function $H_{\mathrm{EFF}}(\mathbf{p})=H_{P}(\mathbf{p})+\varepsilon H_{A}(\mathbf{p})$ and the Eq. (16) can be then rewritten as

$$
\hat{I}(\mathbf{p})=B \delta(\mathbf{p})+\hat{P}(\mathbf{p}) \times H_{\mathrm{EFF}}(\mathbf{p}) .
$$

from which the spectrum of the real part of the scattering potential can be recovered by using a simple deconvolution:

$$
\hat{P}(\mathbf{p})=\frac{\hat{I}(\mathbf{p})-B \delta(\mathbf{p})}{H_{\mathrm{EFF}}(\mathbf{p})} .
$$

However, as in the case of the C-ODT, a more sophisticated deconvolution than Eq. (19) is required for RI reconstruction. For example, in this PC-ODT modality the regularized Wiener deconvolution [31] provides a reliable and fast RI reconstruction from:

$$
\hat{P}(\mathbf{p})=\frac{(\hat{I}(\mathbf{p})-B \delta(\mathbf{p})) H_{\mathrm{EFF}}^{*}(\mathbf{p})}{\left|H_{\mathrm{EFF}}(\mathbf{p})\right|^{2}+\beta},
$$

as experimentally demonstrated in [30]. The regularization parameter $\beta$ depends on the signal to noise ratio (SNR) of the intensity images and typically is within the range $\beta \sim 10^{-2}-10^{-4}$. Then, the scattering potential $V(\mathbf{r})$ is easily calculated by inverse 3D FT of Eq. (20). Since $n^{2}(\mathbf{r})=P(\mathbf{r}) k_{0}^{-2}(1+\mathrm{i} \varepsilon)+n_{m}^{2}$, the RI distribution is finally obtained:

$$
n(\mathbf{r}) \approx n_{\mathrm{re}}(\mathbf{r})=\sqrt{\frac{\left|n^{2}(\mathbf{r})\right|+\operatorname{Re}\left\{n^{2}(\mathbf{r})\right\}}{2}} .
$$

The 3D RI reconstruction process in this PC-ODT modality is summarized in the flowchart displayed in Fig. 3. The first step consists of measuring a stack of images, $I(\mathbf{r})$, obtained by scanning along the optical axis (in this case the $\mathrm{z}$-axis). The deconvolution with the digital camera transfer function is recommended for partial compensation of relatively low contrast images [30]. The next step consists of a background removal that alternatively can be performed in Fourier domain. Then the intensity images are normalized to ensure the energy conservation in the measured stack. The deconvolution Eq. (20) is then performed in the Fourier domain yielding $\hat{P}(\mathbf{p})$ from which the scattering potential $P(\mathbf{r})$ is obtained by calculating the inverse FT. Finally, the 3D RI of the object is recovered from Eq. (21). We recall that the described PC-ODT technique assumes quasi-monochromatic illumination.

Note that the described direct method for recovering of the object 3D RI can be also applied in the case of coherent illumination by using the same illumination scanning process used in C-ODT. Nevertheless, such a variant of C-ODT requires a big amount of data to be collected because for each scanning position in the condenser aperture diaphragm a corresponding stack of intensity images has to be measured, thus turning it into a really time consuming alternative.

By comparing the described schemes for 3D RI recovery (see Fig. 1 and Fig. 3), it seems that the PC-ODT method is simpler than the C-ODT. Indeed, it does not require intermediate recovery of complex-field amplitude and the complicated assembling procedure of the scattering potential spectrum. As a result, PCODT needs less data processing as well as less computation time than the C-ODT counterpart. This makes PC-ODT more suited for almost real-time quantitative 3D and 4D imaging (e.g. see [32]). However, the PC-ODT scheme presented in Fig. 3 assumes a non absorbing sample or the proportionality between the real and imaginary part of scattering potential. Otherwise, an additional stack of intensity distribution acquired for different illumination conditions ( $a(\mid \mathbf{s})$ distribution) is required to obtain $n(\mathbf{r})$ [29]. With reference to the C-ODT method, it allows recovering the real part of the complex scattering potential from the same data set. 
In principle, the C-ODT and the PC-ODT provide access to a similar spatial frequency content of the object. However, the low values of the POTF at $p_{z}=0$ [see Fig. 2(a) and 2(c)] difficult the correct recovery of information from this spatial frequency region for non-absorbing samples, while the proper assembling of synthetic CTF for the C-ODT avoids such a problem. On the other side, the coherent illumination is accompanied by speckle noise and parasitic reflections that in turn are negligible in the PC-ODT framework.

It is also worth pointing out that the missing-cone problem equally affects both ODT modalities. The missing-cone region is smaller for the larger $\mathrm{NA}_{\mathrm{c}}$ and $\mathrm{NA}_{\mathrm{o}}$, however, it can not be completely filled without applying mechanical rotation of the object. The utilization of object rotation [33], multiple angle illumination [23] and even the combined use of both of them [14] have already been experimentally exploited for analysis of static objects. Nevertheless, it results cumbersome for analysis of dynamic objects such as living cells. Some prior assumptions about the object (for instance, a priori knowledge of its shape or imposing the constrain that its RI is higher than the surrounding medium in which is immersed) or an iterative approach for RI recovery can help to compensate the lack of certain spatial frequency information (e.g. see [15]). Such methods can be applied for both ODT modalities. Nevertheless, it can be seen in Fig. 2 that the use of high NAs significantly mitigates the missing-cone problem especially when tiny structures (high spatial frequency values) are analyzed.

\section{EXPERIMENTAL SETUPS FOR ODT IMPLEMENTA- TION}

The experimental implementation of ODT consists of, apart from a microscope, two additional modular setups: one that provides the illumination control over the sample and another one for the measurement of the required intensity distributions. Both setups have to be programmable and fast switchable, which is specially needed when ODT is applied for analysis of dynamic objects such as living cells. This section covers some relevant ODT schemes already settled in the state of the art as well as some new proposals based on modifications in either the illumination or measurement setups.

Nowadays, the modality of interferometric C-ODT is a wellestablished technique often used for 3D RI imaging of biological specimens. Several specially designed commercial holographic microscopes are available for its implementation. A basic scheme of the experimental setup typically required for interferometric C-ODT $[4,8,9]$ is displayed in Fig. 4 . In this case, a collimated laser beam is divided into two components referred to as illumination and reference beams. The illumination beam is redirected by using a turning mirror in order to sequentially scan the back focal plane of the condenser lens. This scan provides the tilted illumination required in C-ODT as it has been previously described. For each scanning position a hologram is recorded by the digital camera. The interferometric setup depicted in Fig. 4 corresponds to an off-axis configuration where a second turning mirror is used to preserve the interference angle between the reference and object beams during the illumination scanning process. In general, hundreds of holograms are required to reconstruct the 3D RI of the object by using the C-ODT algorithm explained in the previous section, see the flowchart sketched in Fig. 1. Note that other DH techniques such as phase-shifting interferometry can be used instead, however, it requires at least four holograms for each scanning position making slower the

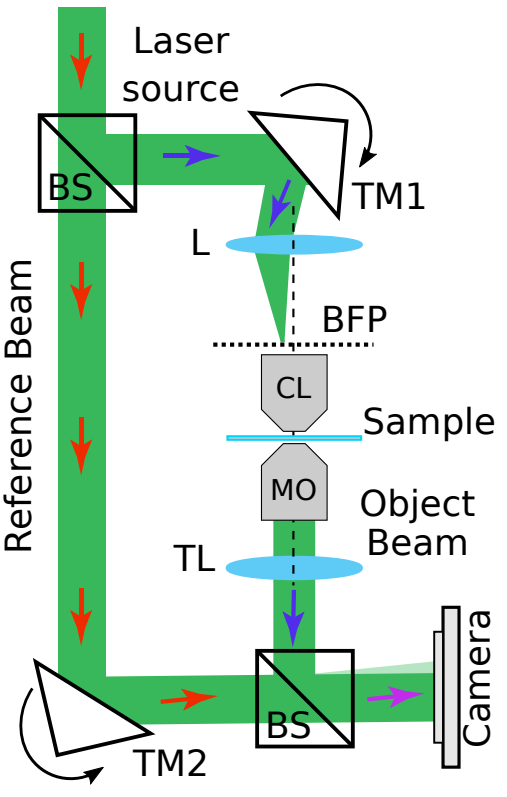

Fig. 4. Sketch of an interferometric setup for implementation of the C-ODT technique in a holographic microscope. The first turning mirror (TM1) controls the illumination scanning process while the second one (TM2) is used to preserve the interference angle between the reference and object beams. Note that an input collimated laser beam is divided by using a beam splitter (BS) while the object and reference beams are recombined by a BS mounted in front of the camera (e.g. sCMOS). A convergent lens (L) is used for focusing the collimated illumination beam onto the back focal plane (BFP) of the condenser lens (CL). The microscope objective lens (MO) collects the laser beam passing by the sample (object beam) at a given scanning position. Then the tube lens (TL) images the object beam into the detector plane of the camera.

C-ODT reconstruction process.

While the explained interferometric C-ODT needs a specific holographic microscope with a laser illumination source, the PCODT is compatible with conventional wide-field microscopes equipped with LED or filtered halogen lamps providing the illumination. For example, the PC-ODT technique developed in [30] uses a quasi-monochromatic LED source to illuminate the whole back focal plane of the condenser lens of a bright-field microscope. Alternatively, the digital light processing (DLP) technology based on DMD (digital mirror device) projectors or LED arrays can be used in the microscope illumination scheme providing fast switchable control of both the light wavelength (red, green and blue LEDs) and illumination coherence [34, 35], see Fig. 5. Thanks to the DLP, it is possible the implementation of both C-ODT and PC-ODT using the same setup. In C-ODT case, the projection of an image in form of small circle (spot) onto the back focal plane of the condenser lens (under Köhler illumination) provides a tilted coherent illumination over the sample at a certain angle defined by the position of such spot. The DLP projector also permits the fast switching of the illumination wavelength if needed. Moreover, the low temporal coherence of the LED source ensures for suppression of the speckle noise.

The intensity measurement module sketched in Fig. 5 can be used for axial image scanning required for the PC-ODT as well as for the C-ODT based on TIE or iterative algorithms, which need a relatively small stack of refocused intensity images for 


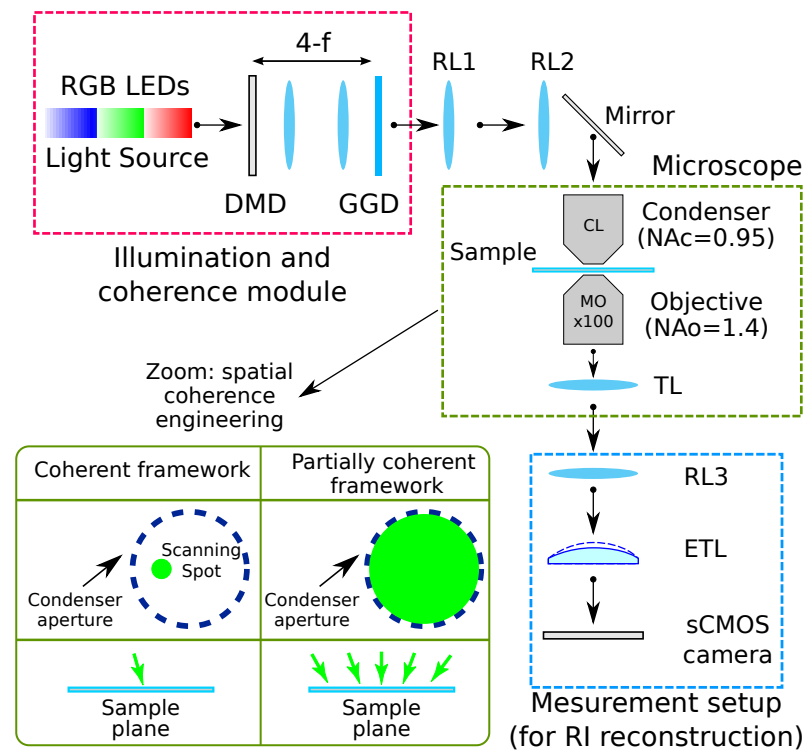

Fig. 5. Sketch of a non-interferometric setup for implementation of both the PC-ODT and C-ODT techniques in a standard wide-field microscope. The illumination system consists of a DLP projector whose projection lens has been replaced by a 4 -f setup to obtain a real image of the DMD display onto a static ground glass diffuser, thus working as a real screen. Such a real image (e.g. the green circle) is then relayed onto the back focal plane of the condenser lens by using two convergent lenses (RL1 and RL2). The refocusing module comprises a relay lens (RL3) and the electrically focus tunable lens (ETL). The required axially scanned images are measured by using a high speed camera (e.g. sCMOS).

phase recovery. In this setup the optical refocusing is achieved by using an electrically focus tunable lens (ETL) incorporated in front of a digital camera [30,35], see Fig. 5, thus avoiding the use of mechanical refocusing with a piezo-stage [29]. In particular, this is beneficial when high NA immerse objectives are used. Specifically, the 3D RI of the sample is reconstructed from the high-speed measurement of a single stack of refocused intensity images [30]. Both the DLP projector and the ETL can be easily incorporated in conventional wide-field microscopes [35] and synchronized between themselves and the digital camera.

Therefore, the aforementioned readily automatized setup can be used for the implementation of both ODT modalities: CODT and the described PC-ODT. Other PC-ODT techniques, like the 3D differential phase contrast approach in which different halves of the condenser aperture diaphragm are sequentially illuminated [29], can benefit from this setup.

\section{PC-ODT: EXPERIMENTAL RESULTS}

The interferometric C-ODT technique has been applied in numerous experimental studies of both inorganic and biological objects along the last decade, see for example $[6-12,19]$. Since the described PC-ODT technique is less known, this section is devoted to illustrate its performance and capabilities in practice. In particular, here we consider the 3D RI reconstruction of diatom cells $\left(n_{s} \sim 1.45-1.46\right)$ immersed in oil $\left(n_{m} \sim 1.518\right.$, Olympus type-F) to deal with the weak object approximation [30]. Specifically, we use the same experimental setup reported in [30] for PC-ODT (similar to Fig. 5), which comprises a quasi-
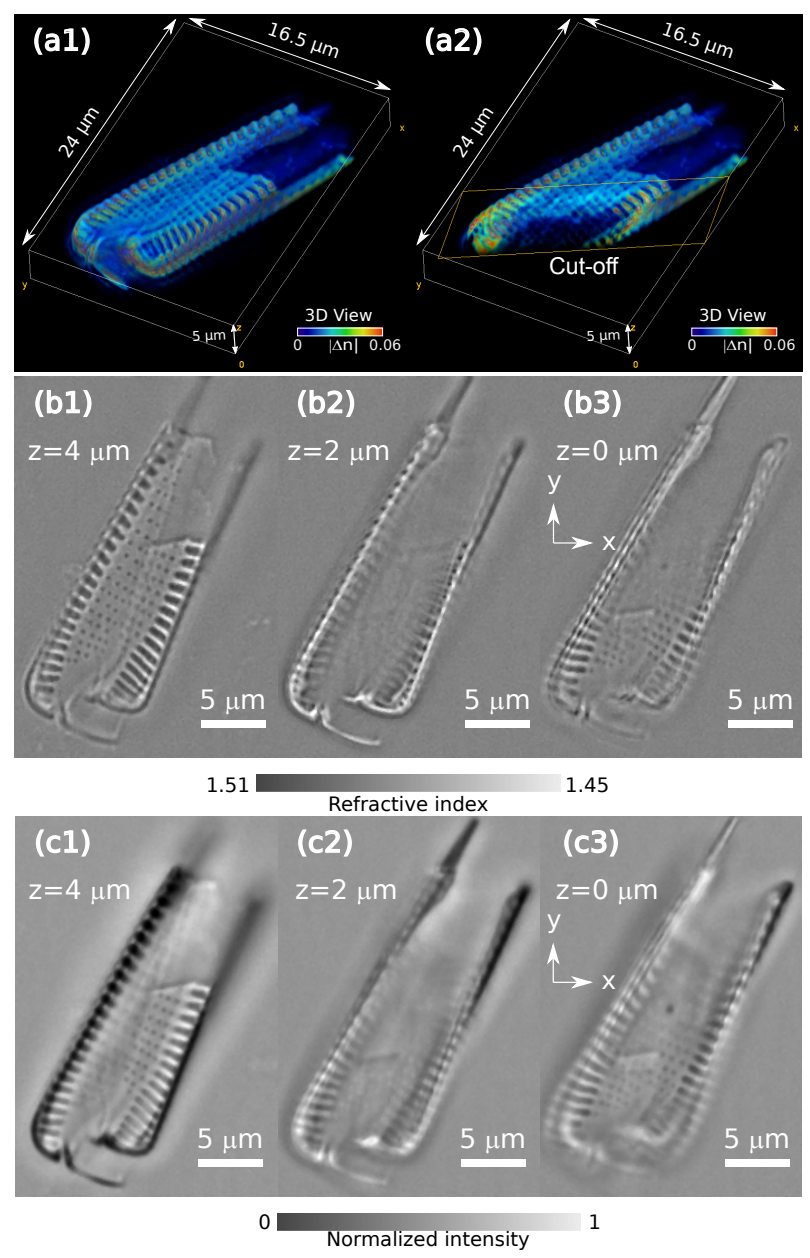

Fig. 6. (a1-a2) Volumetric representation of the reconstructed 3D RI distribution, $\Delta n(\mathbf{r})=\left|\operatorname{Re}\left\{n_{s}(\mathbf{r})\right\}-n_{\mathrm{m}}\right|$, of a diatom by using the PC-ODT. The sample exhibits a clearly observable periodicity of the cell wall indents in the axial cross section. Note that part of the upper diatom wall has been broken. (b1b3) 2D RI slices of in three different XY planes. The observed circular-like pores have a diameter of $\sim 710 \mathrm{~nm}$. (c1-c3) The $2 \mathrm{D}$ intensity slices corresponding to the same RI planes displayed in the figures (b1-b3).

monochromatic LED illumination source $\left(\lambda_{0}=450 \mathrm{~nm}\right)$ together with a high-NA objective lens $\left(\mathrm{NA}_{\mathrm{o}}=1.4\right.$, Olympus UPLSAPO $100 \times$, oil immersion $\left.n_{i m m}=1.518\right)$ and condenser lens $\left(\mathrm{NA}_{\mathrm{C}}=0.95\right)$. The spatial resolution achieved in the traverse direction is $\Delta x=\Delta y=\lambda_{0} /\left(\mathrm{NA}_{\mathrm{o}}+\mathrm{NA}_{\mathrm{c}}\right) \approx 200 \mathrm{~nm}$ while $\Delta z=\lambda_{0} /\left(n_{\text {imm }}-\left(n_{\text {imm }}^{2}-\mathrm{NA}_{\mathrm{o}}^{2}\right)^{1 / 2}\right) \approx 500 \mathrm{~nm}$ in the axial direction. The optical refocusing module (ETL, Optotune EL-10-30-C) and the sCMOS camera (Hamamatsu, Orca Flash 4.0, 16-bit gray-level, pixel size of $6.5 \mu \mathrm{m}$ ) have been synchronized allowing for automatic axial scanning of 50 refocused images $(400 \times 400$ pixels $)$ in a time of $100 \mathrm{~ms}$.

The diatoms are aquatic micro-organisms whose walls (based on silicon dioxide, $n_{s} \sim 1.45-1.46$ ) are typically perforated by multiple tiny pores $(0.1-1 \mu \mathrm{m})$ allowing for the exchange of substances with the environment. The diversity of structural features and small perforations of the diatoms are useful for testing different imaging methods and in particular for the $\mathrm{C}$ ODT (see for example [19]) and PC-OCT techniques. Fig. 6(a1) 
shows a volumetric representation of a diatom, corresponding to its 3D RI distribution $\Delta n(\mathbf{r})=\left|\operatorname{Re}\left\{n_{s}(\mathbf{r})\right\}-n_{\mathrm{m}}\right|$, that have been reconstructed by using PC-OCT. A cross section of the 3D RI distribution of the diatom is also displayed in Fig. 6(a2) in which a cut-off plane is used to visualize the inner part of the diatom. Several 2D slices of the RI in the XY-plane are also displayed in Fig. 6(b1-b3) from which one is able to measure the diameter of the circular-like pores $(\sim 710 \mathrm{~nm})$ as well as the RI value of the cell wall (in the range of $n_{\mathrm{s}}=1.45-1.47$ ), which is in good agreement with those previously reported elsewhere [36]. Note that the RI value is higher near the pores than on other parts of the cell wall because the immersion oil completely filled the holes. As observed in Fig. 6(b1-b3), it is possible to distinguish different cell walls at the bottom $(z=0 \mu \mathrm{m})$ and the top $(z=4 \mu \mathrm{m})$ of the diatom. These walls of the diatom exhibit multiple perforations, unlike the intermediate slices (e.g., $z=$ $2 \mu \mathrm{m}$ ) in which the inner part of the diatom is mostly filled by the surrounding oil medium. The intensity slices corresponding to the Fig. 6(b1-b3) are shown in Fig. 6(c1-c3). Let us underline that the measurement of the stack of intensity images $(400 \times 400 \times 50$ voxels) has been performed in $100 \mathrm{~ms}$ with an axial step of $250 \mathrm{~nm}$ (optical refocusing). These promising results indicate that PC-ODT can be a competitive alternative to the traditional C-ODT techniques, making possible label-free 3D quantitative imaging for almost real-time applications.

\section{CONCLUSIONS}

Throughout this article, a comparison between two modalities of ODT (either based on using fully or partially coherent illumination over the sample) has been drawn. Both methods provide reliable 3D RI reconstruction of transparent objects under the weak scattering and low absorption approximations. Nevertheless, these techniques are conceptually and experimentally different. In particular, the widely applied interferometric C-ODT is based on an illumination scanning method required to collect all the accessible spatial frequency content of the object. In contrast, the PC-ODT technique accesses to a similar spatial frequency content by axial scanning of intensity images of the object, thus speeding up the 3D RI reconstruction. Another important difference lies in the hardware needed to apply them since PC-ODT only requires adding a refocusing system (e.g. an electrically focus tunable lens mounted in front of the camera) to a conventional microscope, whereas the commonly used interferometric C-ODT needs a specifically designed holographic microscope comprising an off-axis interferometric setup (including several galvanometer turning mirrors or acousto-optic modulators) that is more expensive. Regarding the data processing, the PC-ODT is simpler than the coherent counterpart because C-ODT applies a complicated and computational demanding algorithm to put together all the frequency content components of the object, which is unnecessary in PC-ODT. Moreover, the PC-ODT technique is free of speckle noise. However, the PC-ODT has downsides that need to be taken into account: while the C-ODT allows recovering of both the real and imaginary parts of the $\mathrm{RI}$, the imaging of the complex RI with PC-ODT requires an additional stack of intensity measurements under different illumination conditions or some priors. In general, the C-ODT and the PC-ODT have access to the same spatial frequency content. Nevertheless, the proper assembled synthetic CTF is more uniform (that is well suited for the deconvolution operation in C-ODT) than the OTFs obtained in PC-ODT for fully opened condenser diaphragm. Here the first-order Born approximation has been considered, however, the C-ODT method can be easily adapted to Rytov approximation. In the case of PC-ODT the Rytov approximation has not been developed yet.

Finally, here we have introduced an experimental setup compatible with both non-interferometric C-ODT and PC-ODT. We believe that it can be useful for a fair experimental comparison between both techniques using the same test sample that allows further clarifying their advantages and limitations. The compatibility of the proposed setup with conventional wide-field microscope allows easily switching from both ODT modalities and might complement each other.

\section{FUNDING INFORMATION}

The Spanish Ministerio de Economía y Competitividad is acknowledged for funding the project TEC2014-57394-P.

\section{REFERENCES}

1. E. Wolf, "Three-dimensional structure determination of semitransparent objects from holographic data," Opt. Commun. 1, 153-156 (1969).

2. R. Dandliker and K. Weiss, "Reconstruction of the three-dimensional refractive index from scattered waves," Opt. Commun. 1, 323 - 328 (1970).

3. F. Charrière, A. Marian, F. Montfort, J. Kuehn, T. Colomb, E. Cuche, P. Marquet, and C. Depeursinge, "Cell refractive index tomography by digital holographic microscopy," Opt. Lett. 31, 178 (2006).

4. W. Choi, C. Fang-Yen, K. Badizadegan, S. Oh, N. Lue, R. Dasari, and M. Feld, "Tomographic phase microscopy," Nat. Methods 4, 717-720 (2007).

5. O. Haeberlé, K. Belkebir, H. Giovaninni, and A. Sentenac, "Tomographic diffractive microscopy: basics, techniques and perspectives," J. Mod. Opt 57, 686-699 (2010).

6. Y. Sung, W. Choi, C. Fang-Yen, K. Badizadegan, R. R. Dasari, and M. S. Feld, "Optical diffraction tomography for high resolution live cell imaging." Opt. Express 17, 266-277 (2009).

7. Y. Cotte, F. M. Toy, C. Arfire, S. S. Kou, D. Boss, I. Bergoënd, and C. Depeursinge, "Realistic 3D coherent transfer function inverse filtering of complex fields." Biomed. Opt. Express 2, 2216-30 (2011).

8. Y. Cotte, F. Toy, P. Jourdain, and N. Pavillon, "Marker-free phase nanoscopy," Nat. Photonics 7, 113-117 (2013).

9. K. Kim, K. Kim, H. Park, J. Ye, and Y. Park, "Real-time visualization of 3-D dynamic microscopic objects using optical diffraction tomography," Opt. Express 21, 32269-32278 (2013).

10. Y. Kim, H. Shim, K. Kim, H. Park, and J. Heo, "Common-path diffraction optical tomography for investigation of three-dimensional structures and dynamics of biological cells," Opt. Express 22, 228-232 (2014).

11. K. Kim, J. Yoon, and Y. Park, "Simultaneous 3D visualization and position tracking of optically trapped particles using optical diffraction tomography," Optica 2, 343-346 (2015).

12. J. Jung, K. Kim, J. Yoon, and Y. Park, "Hyperspectral optical diffraction tomography," Opt. Express 24, 2006 (2016).

13. S. Vertu, J.-J. Delaunay, I. Yamada, and O. Haeberlé, "Diffraction microtomography with sample rotation: influence of a missing apple core in the recorded frequency space," Open Physics 7, 22-31 (2009).

14. B. Simon, M. Debailleul, M. Houkal, C. Ecoffet, J. Bailleul, J. Lambert, A. Spangenberg, H. Liu, O. Soppera, and O. Haeberlé, "Tomographic diffractive microscopy with isotropic resolution," Optica 4, 460-463 (2017).

15. J. Lim, K. Lee, K. H. Jin, S. Shin, S. Lee, Y. Park, and J. C. Ye, "Comparative study of iterative reconstruction algorithms for missing cone problems in optical diffraction tomography." Opt. Express 23, 16933-48 (2015).

16. U. S. Kamilov, I. N. Papadopoulos, M. H. Shoreh, A. Goy, C. Vonesch, M. Unser, and D. Psaltis, "Learning approach to optical tomography," Optica 2, 517-522 (2015). 
17. A. Berdeu, F. Momey, B. Laperrousaz, T. Bordy, X. Gidrol, J.-M. Dinten, N. Picollet-D'hahan, and C. Allier, "Comparative study of fully threedimensional reconstruction algorithms for lens-free microscopy," Appl. Opt. 56, 3939-3951 (2017).

18. V. Lauer, "New approach to optical diffraction tomography yielding a vector equation of diffraction tomography and a novel tomographic microscope," J. Microsc 205, 165-176 (2002).

19. M. Debailleul, B. Simon, V. Georges, O. Haeberlé, and V. Lauer, "Holographic microscopy and diffractive microtomography of transparent samples," Meas. Sci. Technol 19, 074009 (2008).

20. K. Kim, H. Yoon, M. Diez-Silva, M. Dao, R. R. Dasari, and Y. Park, "Highresolution three-dimensional imaging of red blood cells parasitized by Plasmodium falciparum and in situ hemozoin crystals using optical diffraction tomography," J. Biomed. Opt 19, 011005 (2013).

21. T. Kim, R. Zhou, L. L. Goddard, and G. Popescu, "Solving inverse scattering problems in biological samples by quantitative phase imaging," Laser Photon. Rev. 10, 13-39 (2016).

22. M. Born and E. Wolf, Principles of Optics (Cambridge University Press, Cambridge, 1999).

23. M. H. Jenkins and T. K. Gaylord, "Three-dimensional quantitative phase imaging via tomographic deconvolution phase microscopy," Appl. Opt. 54, 9213 (2015).

24. Y. Bao and T. K. Gaylord, "Quantitative phase imaging method based on an analytical nonparaxial partially coherent phase optical transfer function," J. Opt. Soc. Am. A 33, 2125 (2016).

25. A. J. Devaney, "A filtered backpropagation algorithm for diffraction tomography," Ultrasonic imaging 4, 336-350 (1982).

26. J. Kostencka, T. Kozacki, A. Kuś, and M. Kujawińska, "Accurate approach to capillary-supported optical diffraction tomography," Opt. Express 23, 7908-7923 (2015).

27. P. Müller, M. Schürmann, and J. Guck, "The theory of diffraction tomography," arXiv preprint arXiv:1507.00466 (2015).

28. N. Streibl, "Three-dimensional imaging by a microscope," JOSA A 2 , 121-127 (1985).

29. M. Chen, L. Tian, and L. Waller, "3D differential phase contrast microscopy," Biomed. Opt. Express 7, 3940-3950 (2016).

30. J. M. Soto, J. A. Rodrigo, and T. Alieva, "Label-free quantitative 3D tomographic imaging for partially coherent light microscopy," Opt. Express 25, 15699 (2017).

31. D. S. C. Biggs, "3D deconvolution microscopy," Curr. Protoc. Cytom. pp. $1-20(2010)$

32. J. A. Rodrigo, J. M. Soto, and T. Alieva, "Fast label-free microscopy technique for $3 d$ dynamic quantitative imaging of living cells," Biomed. Opt. Express 8, 5507-5517 (2017).

33. A. Kuś, M. Dudek, B. Kemper, M. Kujawińska, and A. Vollmer, "Tomographic phase microscopy of living three-dimensional cell cultures." J. Biomed. Opt. 19, 046009 (2014).

34. E. C. Samson and C. M. Blanca, "Dynamic contrast enhancement in widefield microscopy using projector-generated illumination patterns," New J. Phys. 9, 363-363 (2007).

35. J. A. Rodrigo and T. Alieva, "Illumination coherence engineering and quantitative phase imaging." Opt. Lett. 39, 5634-7 (2014).

36. S. Yamanaka, R. Yano, H. Usami et al., "Optical properties of diatom silica frustule with special reference to blue light," J. Appl. Phys 103, 074701 (2008). 